\title{
Avances en la construcción del Inventario de Estigma relacionado con el VIH (IE-VIH) en Buenos Aires, Argentina
}

\author{
Pablo David Radusky ${ }^{1}$, Virginia Zalazar ${ }^{2}$, Inés Arístegui ${ }^{3}$, Omar Sued ${ }^{4}$ y Isabel María \\ Mikulic $^{5}$
}

\begin{abstract}
RESUMEN
La expresión del estigma relacionado con el VIH, siendo culturalmente determinada, ha sufrido cambios a través de las décadas, asumiendo formas más sutiles, aunque igualmente perjudiciales. Por consiguiente, con el fin de medir este constructo, deben ser desarrollados nuevos instrumentos más actualizados y que capturen sus particularidades en contextos específicos. Tres dimensiones del estigma son reconocidas por los principales marcos teóricos: internalizado, anticipado y experimentado. Los objetivos de este estudio fueron explorar el funcionamiento de un conjunto inicial de items de un nuevo instrumento para medir estigma, obtener evidencia de confiabilidad y validez del instrumento y describir los niveles de estigma en personas con VIH adultas del Área Metropolitana de Buenos Aires (AMBA). Cinco expertos evaluaron la claridad, relevancia y validez de contenido de 88 reactivos preliminares. Una versión depurada de 77 items fue administrada a una muestra piloto. El análisis estadístico incluyó el análisis descriptivo de los items, alfas de Cronbach para establecer la confiabilidad y correlaciones con la Escala de Estigma de VIH, para verificar la validez de criterio. La muestra final consistió en 46 personas con VIH, adultas, residentes en el AMBA (67\% hombres, $31 \%$ mujeres y $2 \%$ mujeres transgénero). La media de edad fue de 41 años $(D E=10.68)$. Cincuenta ítems con adecuada asimetria, curtosis y correlación ítem-total fueron conservados. Las escalas mostraron buena confiabilidad: Internalizado $=.86$; Anticipado $=.85$ y Experimentado $=.86$. Cada escala correlacionó positiva y significativamente con su criterio. Se hallaron niveles más elevados de estigma anticipado, que de estigma internalizado y experimentado. Este nuevo instrumento diseñado para medir estigma relacionado con el VIH considerando sus particularidades locales, ha demostrado adecuada confiabilidad y validez, en esta primera etapa de su construcción. La etapa siguiente consistirá en un análisis factorial con una muestra de mayor tamaño con el fin de incrementar la evidencia de validez.
\end{abstract}

Palabras clave: VIH, estigma, medición, construcción.

\section{Progress in the construction of the Stigma related to HIV Inventory (SI- HIV)}

\begin{abstract}
The expression of stigma related to HIV is culturally determined and has changed through decades, assuming subtler, but still harmful forms. Therefore, aiming at assessing this construct, updated instruments that capture its particularities in specific contexts should be designed. Most theoretical frameworks recognize three dimensions of stigma: internalized, anticipated and enacted. The objectives of this study were: to explore an initial set of items of a new measurement of stigma, to obtain evidence of reliability
\end{abstract}

\footnotetext{
1 y 5 Facultad de Psicología, Universidad de Buenos Aires, Argentina; pablodradusky@yahoo.com.ar

2 y 4 Fundación Huésped, Argentina.

${ }^{3}$ Fundación Huésped, Universidad de Palermo, Argentina.
} 
and validity, and to describe the levels of stigma among adults with HIV from Buenos Aires city. Five experts evaluated the clarity, relevance and content validity of 88 preliminary items. A depurated version of 77 items was administered to a pilot sample. The statistical analysis included a descriptive item analysis, Cronbach's alphas to evaluate reliability and, to establish criterion-related validity, correlations with the HIV Stigma Scale. The final sample consisted of 46 individuals with HIV $(67 \%$ men, 31\% women and $2 \%$ transgender women). Mean age was 41 years $(S D=10.68)$. Fifty items with adequate skewness, kurtosis and item-total correlation were retained. Scales showed good reliability: Internalized $=.86$; Anticipated $=.85$ and Enacted $=.86$ ). Each scale significantly and positively correlated with its criterion. Higher levels of anticipated stigma were observed, followed by internalized and enacted stigma. This newly developed instrument, designed to measure HIV stigma considering local particularities, demonstrated adequate validity and reliability in the first stage of its construction. The following step will consist of a factor analysis with a larger sample to increase evidence of validity.

Keywords: HIV, stigma, measurement, construction.

El estudio del estigma tiene sus raíces en las teorizaciones de Erving Goffman (1963) quien ofreció una de las primeras conceptualizaciones de este fenómeno como "un atributo que es profundamente desprestigiante" (p. 13) y que reduce al portador de ser una persona total y corriente a una "manchada" y menospreciada. El resultado es una "identidad deteriorada" o arruinada. Este planteamiento teórico inicial dio un fuerte impulso al estudio del estigma en el campo de la Psicología Social y la investigación dentro de esta disciplina proliferó desde un enfoque socio-cognitivo (Parker \& Aggleton, 2003). Este abordaje pone el énfasis en las percepciones individuales y sus consecuencias en las interacciones sociales, ubicando el origen del estigma en la cognición (Link \& Phelan, 2001; Mahajan et al., 2008).

En años recientes, numerosos investigadores han criticado este enfoque por considerar que pone un acento excesivo en el plano individual, mientras descuida las condiciones estructurales y contextuales que generan estigma y exclusión de la vida social (Mahajan et al., 2008; Ogden \& Nyblade, 2005). Este enfoque alternativo, denominado estructural, propone redefinir el estigma como un proceso social que se produce en el marco de relaciones de poder desiguales entre grupos (Link \& Phelan, 2001; Parker \& Aggleton, 2003).

Desde esta concepción estructural, Link y Phelan $(2001,2006)$ definen al estigma como la ocurrencia simultánea de los siguientes componentes:

- Distinción y etiquetamiento de diferencias: toda sociedad identifica qué atributos son socialmente importantes y cuáles no lo son, en un proceso de selección social, y produce categorias para etiquetar las diferencias socialmente relevantes;

- Estereotipación: algunas etiquetas y categorias son asociadas a estereotipos que implican atributos negativos o socialmente indeseables;

- Separación "ellos" - "nosotros": aquellos que comparten la etiqueta son vistos como esencial y particularmente diferentes a los que no la poseen. De este modo, se genera una distancia o alejamiento simbólico y real entre ambos grupos;

- Pérdida de estatus y discriminación: la separación ubica a quienes poseen la etiqueta en una posición inferior respecto del otro grupo, dado que el atributo aplicado está asociado a estereotipos o características negativas y socialmente indeseables. En 
consecuencia, se produce una racionalización que legitima el menosprecio, el rechazo y la exclusión de los primeros.

La irrupción del VIH, en el año 1981, agregó un elemento más al listado de atributos socialmente estigmatizables y estigmatizados. La rápida asociación entre estigma y VIH se debió a una multiplicidad de motivos. En primer lugar, es posible mencionar la peligrosidad y el riesgo socialmente percibido respecto de la infección. Según Jones et al. (1984), el estigma es mayor cuando el atributo es percibido como peligroso para los demás, activando una de las funciones sociales del estigma: la evitación de la enfermedad (Kurzban \& Leary, 2001; Phelan, Link \& Dovidio, 2008).

Sin embargo, también influyó el hecho de que en un inicio la epidemia afectara predominantemente a ciertos grupos que, por sus prácticas y atributos, ya eran previamente estigmatizados: hombres gays, personas transgénero, usuarios de drogas y hombres y mujeres que ejercian la prostitución, entre otros (Bilder, 2010; Pecheny, Manzelli \& Jones, 2007). En este sentido, el estigma relacionado con el VIH vino a asentarse en una estructura preexistente de prejuicios sociales relacionados con la orientación sexual, la identidad de género, la promiscuidad, el uso de drogas ilegales, la prostitución y la pobreza (Deacon, 2006; Mahajan et al., 2008; Pecheny, 2008; Pecheny et al., 2007), todos atributos en sí mismos estigmatizados. Esta interacción entre el estigma relacionado con el VIH y estigmas preexistentes ha sido denominada estigma "compuesto" o "en capas" (Nyblade, 2006). Este concepto remite a otros enfoques teóricos ya ampliamente desarrollados en las ciencias sociales, como el de interseccionalidad (Bauer, 2014; Crenshaw, 1989). Y es una de las razones principales por las que el estigma relacionado con el VIH es tan fuerte (Earnshaw, Smith, Chaudoir, Rivet Amico \& Copenhaver, 2013; Earnshaw, Lang, Lippitt, Jin \& Chaudoir, 2015; Nyblade, 2006).

Tomando como base la conceptualización general de Link y Phelan (2001) e integrando los aportes de otros autores (Berger, Ferrans \& Lashley, 2001; Deacon, 2006; Earnshaw et al., 2013; Hatzenbuehler, Phelan \& Link, 2013; Herek, Capitanio \& Widaman, 2002; Link \& Phelan, 2006; Ogden \& Nyblade, 2005), es posible definir al estigma relacionado con el VIH como la ocurrencia simultánea, en el marco de relaciones sociales de poder, de etiquetamiento, estereotipación y separación grupal, acompañados de emocionalidad negativa, dirigida a personas por el hecho de vivir con VIH. Esto ocurre debido a la construcción del VIH como una marca desprestigiante o indeseable o un atributo negativamente valorado, en un contexto socio-cultural dado, que resulta en devaluación y descrédito de la identidad, pérdida de estatus y no aceptación social plena, desventaja social de las personas con VIH y, finalmente, discriminación, rechazo y exclusión de las mismas.

Particularmente, se han identificado dos elementos que originan y sostienen el estigma relacionado con el VIH: las actitudes negativas hacia los grupos más expuestos o afectados (componente afectivo-moral) y la falta de conocimiento que genera temor al contacto y la transmisión casual (componente cognitivo-informacional) (Herek et al., 2002; Ogden \& Nyblade, 2005). Ogden y Nyblade (2005) han hipotetizado que ambos componentes constituyen el núcleo básico de este tipo de estigma que trasciende los diversos contextos culturales particulares.

Las actitudes negativas implican un juicio moral producto de dos elementos: a) la percepción de que la infección por VIH es un atributo 
controlable o prevenible, por lo que las personas que viven con él son, en mayor o menor medida, culpables por su situación (Crocker, Major \& Steele, 1998; Herek et al., 2002; Ogden \& Nyblade, 2005; Rosas \& Gomes, 2008), y b) la percepción de que las personas con VIH han transgredido normas y valores sociales (Deacon, 2006; Mahajan et al., 2008; Pecheny et al., 2007). Esto permite explicar, en parte, la rápida asociación entre estigma y VIH desde etapas tempranas de la epidemia. Los grupos más afectados desde un inicio ya eran depositarios de estigma social por tener prácticas que diferian de la norma social. Incluso esas mismas prácticas eran las que incrementaban su exposición a la infección, por lo que fueron percibidos como culpables por su destino y por contribuir a la expansión de la epidemia en los distintos países.

Earnshaw et al. (2013) identificaron y diferenciaron tres mecanismos en los que opera psicológicamente el estigma relacionado con el $\mathrm{VIH}$, en un modelo integrador que denominaron "Marco de Estigma relacionado con el VIH". En primer lugar, es posible distinguir un estigma experimentado, constituido por las experiencias efectivas y concretas de discriminación, estereotipación, prejuicio o cualquier forma negativa de distinción, exclusión o restricción, producto de la interacción con individuos o instituciones sociales, que afecten a un sujeto por el hecho de vivir con VIH (Earnshaw et al., 2013; Jeffries et al., 2015; Kipp et al., 2015). En segundo lugar, estos autores reconocen un estigma internalizado, que es la aceptación y adopción dentro del autoconcepto, por parte de las personas con VIH, de los estereotipos, prejuicios, creencias, juicios y sentimientos negativos que la sociedad tiene hacia ellas. Esto produce sentimientos de culpa, vergüenza y desvalorización, autodescalificación y aceptación de que son responsables por su situación y merecen ser tratados negativa e inequitativamente (Berger et al., 2001; Earnshaw et al., 2013; Nyblade, 2006). Finalmente, el tercer mecanismo reconocido es el estigma anticipado, conceptualizado como la creencia, temor o expectativa de que se experimentará prejuicio o discriminación en el futuro, si las otras personas supieran que uno vive con VIH. Está relacionado con la propia conciencia o percepción de la visión negativa de la sociedad acerca de las personas con VIH (Earnshaw et al., 2013; Jeffries et al., 2015; Kipp et al., 2015).

De acuerdo con Earnshaw et al. (2013), estos mecanismos son relativamente independientes y producen efectos diferenciales en la salud y calidad de vida de las personas con VIH. En consonancia con esta conclusión, existe un conjunto nutrido de investigaciones que han demostrado consistentemente las consecuencias negativas del estigma relacionado con el $\mathrm{VIH}$ en la salud y bienestar de las personas con VIH y en los esfuerzos preventivos y asistenciales para contener la epidemia (Baral, Logie, Grosso, Witz \& Beyrer, 2013; Chambers et al., 2015; Herek, Saha \& Burack, 2013; Logie \& Gadalla, 2009). El estigma entorpece las acciones preventivas, obstaculiza el acceso al testeo y atención y reduce la adherencia al tratamiento antirretroviral. De forma directa, resulta un estresor crónico con impacto inmediato en la salud de las personas con VIH (Earnshaw et al., 2015; Hatzenbuehler et al., 2013).

En el contexto argentino, en particular, las investigaciones que describen el nivel de estigma vivido o percibido por las personas con VIH son escasas. E1 antecedente más reciente es un estudio realizado por Fundación Huésped (2012) que reveló que al menos el 59\% de los participantes había experimentado situaciones concretas de estigma (especialmente 
murmuraciones y exclusión de actividades cotidianas) debido al VIH, a otro atributo (como por ejemplo ser homosexual, transgénero o usuario de drogas), o a la combinación de ambos. Sin embargo, la proporción de aquellos que reconocian expectativa y temor de ser estigmatizados (estigma anticipado) y autoestigma (estigma internalizado) ascendió al 94\%. Se concluyó que el estigma interno (internalizado y anticipado) superaba ampliamente al estigma externo (efectivamente experimentado o vivido).

\section{Evaluación del estigma relacionado con el VIH}

La psicología ha producido diversos instrumentos que permiten evaluar y medir el estigma relacionado con el VIH. La mayor parte de ellos son pruebas psicométricas que adoptan el formato de inventarios y autorreportes (Berger et al., 2001; Bunn, Solomon, Miller \& Forehand, 2007; Franke et al., 2014; Kipp et al., 2015; Molina \& Ramirez-Valles, 2013; Visser, Kershaw, Makin \& Forsyth, 2008). Las dimensiones del estigma relacionado con el VIH que son consideradas por cada uno de estos instrumentos son altamente variables. De todos modos, ninguno de ellos incluye y refleja los tres mecanismos del "Marco de Estigma relacionado con el VIH" (Earnshaw et al., 2013) de forma integral. De hecho, Nyblade (2006), ha criticado las medidas construidas hasta el momento para evaluar el estigma relacionado con el VIH, y menciona que estas raramente incorporan todas las dimensiones de este constructo. En particular, resalta dos que aún no han sido adecuadamente consideradas ni integradas en los instrumentos: el estigma experimentado y el estigma compuesto o "en capas".

Adicionalmente, estos inventarios no están disponibles en Argentina, dado que no se han hecho adaptaciones locales. Este vacío refleja la necesidad de contar con instrumentos válidos y confiables, adecuados a nuestro medio y basados en modelos teóricos robustos y actuales, que permitan medir y evaluar el estigma relacionado con el VIH.

Sin embargo, existen motivos para considerar la necesidad de diseñar un nuevo instrumento local en lugar de adaptar aquellos construidos en otros contextos. En primer lugar, se debe tener en cuenta que la expresión del estigma está fuertemente influida por el contexto social y cultural y por el momento histórico particular (Visser et al., 2008). A pesar de que Ogden y Nyblade (2005) han postulado un núcleo común en el estigma relacionado con el VIH que trasciende contextos específicos, las formas en que este fenómeno se vuelve concreto en la vida cotidiana de las personas sí están atravesadas por particularidades culturales y sociales. Las concepciones locales acerca de la sexualidad y la diversidad sexual (homosexualidad y personas transgénero), el nivel de religiosidad de una comunidad, la existencia de campañas preventivas que provean de información sobre el VIH a la población, el grado de avance de la epidemia, entre otros elementos, pueden influir y moderar los niveles de estigma, impactando en las formas específicas en que este se expresa. En este sentido, el muestreo de conductas e indicadores considerados para operacionalizar el fenómeno debe ser representativo del contexto específico de aplicación futura. De esto se desprende la necesidad de desarrollar nuevos instrumentos para medir el estigma relacionado con el VIH, contextualizados, culturalmente significativos para los sujetos que los responden y, por lo tanto, útiles en el entorno en el que fueron gestados (Visser et al., 2008). 
Asimismo, la evolución del estigma relacionado con el VIH ha acompañado la evolución histórica de la epidemia misma. Los cambios drásticos en el curso de la epidemia de VIH, producidos por los avances en la ciencia médica respecto del tratamiento y la prevención, han impactado en la manera en que el estigma se expresa, haciendo que este asuma formas más sutiles, pero igualmente perjudiciales (Herek et al., 2002). En este sentido, a lo largo de tres décadas de epidemia, se ha observado una reducción de las formas más abiertas de estigma (por ejemplo, apoyo a politicas coercitivas, como la cuarentena), mientras que formas más encubiertas (por ejemplo, incomodidad en el contacto con una persona con VIH) aún persisten (Herek et al., 2002). Por esta razón, también es necesario generar instrumentos más actuales, no solo específicos para un contexto sino también para un momento histórico (Visser et al., 2008). A este respecto, las nuevas herramientas de medición deberian reflejar las formas en que el estigma relacionado con el VIH se expresa o es experimentado en la cotidianeidad y en la actualidad.

Como resultado de lo anteriormente expuesto, y dado el vacío de instrumentos locales que permitan medir este fenómeno, se inició el proceso de construcción de un instrumento psicométrico para medir estigma relacionado con el VIH en personas con VIH adultas del Área Metropolitana de la Ciudad de Buenos Aires (AMBA). En el presente artículo se exponen los resultados de la primera etapa de este proceso y se presenta la versión preliminar del instrumento, obtenida luego de la fase de piloto. Esta primera etapa tuvo como objetivos explorar el funcionamiento de un conjunto inicial de items con el fin de arribar a una versión preliminar del instrumento, obtener evidencia de confiabilidad y validez para dicha versión y describir los niveles de estigma relacionado con el VIH en una muestra de personas con VIH adultas del AMBA.

\section{MÉTODO}

\section{Revisión de la literatura y preparación de la versión preliminar}

La construcción del Inventario de Estigma relacionado con el VIH (IE$\mathrm{VIH}$ ) fue realizada de acuerdo con las sugerencias metodológicas de Hogan (2004) para la construcción de pruebas. De esta forma, involucró las siguientes etapas:

\section{a. Revisión de la literatura y operacionalización del constructo}

En primera instancia, se llevó a cabo una búsqueda y revisión bibliográfica exhaustiva de los principales marcos teóricos que contribuyeron a la conceptualización del estigma en general, y del estigma relacionado con el $\mathrm{VIH}$, en particular, con el fin de delimitar teóricamente el constructo. Asimismo, se efectuó una recopilación de los instrumentos psicométricos más recientes construidos para medirlo y disponibles en la literatura.

La definición de estigma brindada por Link y Phelan (2001) fue considerada la más completa y precisa en su formulación, englobando a la mayor parte de las definiciones de otros autores. Además, resultó la conceptualización más frecuentemente citada en los estudios sobre estigma publicados. Por este motivo, fue tomada como referencia para la definición operacional del constructo. Sin embargo, dado que es una definición general de estigma, independiente de atributos estigmatizados particulares, fue 
complementada y enriquecida con aportes de otros autores para adaptarla a la especificidad del VIH (Berger et al., 2001; Deacon, 2006; Earnshaw et al., 2013; Herek et al., 2002; Link \& Phelan, 2006; Ogden \& Nyblade, 2005).

Por otro lado, se analizaron las dimensiones consideradas por los diversos marcos teóricos como componentes del estigma relacionado con el VIH. A pesar de que existen discrepancias entre las distintas conceptualizaciones, se concluyó que todas las propuestas coinciden o pueden ser reducidas teóricamente a las tres dimensiones, sintetizadas en el "Marco de Estigma relacionado con el VIH": estigma experimentado, estigma anticipado y estigma internalizado (Earnshaw et al., 2013). En conclusión, se adoptó este modelo teórico tridimensional para operacionalizar el constructo. Las definiciones finales de estigma relacionado con el VIH y de cada una de sus dimensiones fueron previamente desarrolladas en la Introducción.

\section{b. Preparación de los reactivos}

Se redactó un conjunto preliminar de 88 ítems con el propósito de operacionalizar cada una de las tres dimensiones consideradas. El contenido de los enunciados fue tomado de tres fuentes:

- Entrevistas a personas con VIH: se realizaron 15 entrevistas en profundidad a personas con VIH con el fin de conocer la experiencia y percepción de estigma relacionado con el VIH en el contexto de Argentina $\mathrm{y}$, más específicamente, del Área Metropolitana de Buenos Aires (AMBA). Algunos reactivos están inspirados en frases extraídas del relato de los entrevistados acerca de episodios de estigma vividos por ellos mismos o por otras personas cercanas.

- Otros instrumentos que miden el mismo constructo: Algunos enunciados incluidos en otros instrumentos reconocidos que evalúan el mismo constructo fueron tomados como referencia para la redacción de algunos de los items preliminares. Los instrumentos considerados fueron: HIV Stigma Scale (Sowell et al., 1997), HIV Stigma Scale (Berger et al., 2001), HIV/AIDS Stigma Instrument - PLWA (HASI-P, Holzemer et al., 2007), Internalized Stigma Scale (Sayles et al., 2008), HIV Related Stigma Scales (Visser et al., 2008), HIV/AIDS Stigma Scale para Latinos GBT (Molina \& Ramirez-Valles, 2013) y Van Rie HIV/AIDS-Related Stigma Scale (Kipp et al., 2015). Particularmente, se consideraron aquellos items que funcionaban psicométricamente bien en sus instrumentos de origen, que eran teóricamente muy representativos de la dimensión a la que correspondian y/o describian situaciones que habian sido mencionadas por las personas entrevistadas como habituales en el contexto del AMBA.

- El marco teórico adoptado: finalmente, algunos ítems fueron especialmente redactados para abarcar algún aspecto de la definición de las dimensiones que no se encontraba representado por ninguno de los enunciados tomados de las dos fuentes anteriores.

Para la redacción de los reactivos se siguieron las recomendaciones de Matesanz Nogales (1997) en lo que respecta a claridad, sencillez y comprensión de los items. Con el fin de evitar la aquiescencia se redactaron items directos e inversos. Asimismo, se redactó una consigna general para el 
protocolo y se propuso una escala de respuesta de 4 opciones de 0 (muy en desacuerdo) a 3 (muy de acuerdo).

\section{c. Análisis por juicio de expertos}

Se realizó un juicio de expertos con el propósito de evaluar la calidad de los ítems y la validez de contenido, siguiendo los pasos y criterios recomendados por Escobar-Pérez y Cuervo-Martínez (2008). Tomando la sugerencia de estas autoras, se convocó a 5 especialistas en VIH y estigma: tres psicólogos con experiencia en intervención e investigación con personas con VIH y dos miembros de una organización no gubernamental dedicada a la atención de personas con VIH (uno de ellos era una persona con VIH con experiencia en coordinación de grupos de pares y el restante, un activista de reconocida trayectoria desde el inicio de la epidemia).

Los jueces expertos recibieron entrenamiento para la realización del juicio, especialmente aquellos que no eran psicólogos ni contaban con experiencia en investigación. A cada juez se le proveyó de una planilla confeccionada específicamente para este fin, donde se encontraban detallados los siguientes puntos: 1) instrucciones para la realización del juicio y objetivo del mismo, 2) finalidad de la prueba a construir, 3) población a la que está dirigida la prueba y 4) modo de obtención e interpretación de las puntuaciones (identificando ítems inversos). Asimismo, se incluyó la definición del constructo y de cada dimensión para posibilitar la evaluación de la validez de contenido.

Se solicitó a los expertos que juzgaran y evaluaran el grado de adecuación de cada ítem a cada uno de los 4 criterios propuestos por EscobarPérez y Cuervo-Martínez (2008): coherencia con la definición conceptual, relevancia, claridad y suficiencia (esta última, respecto del conjunto de items de una dimensión y el grado en que todos los aspectos de la definición correspondiente estaban cubiertos por las frases propuestas). De forma adicional, se agregó un quinto criterio, que se denominó representatividad, con el objetivo de garantizar que los items describieran situaciones representativas o significativas para las personas con VIH o realmente frecuentes en su cotidianeidad y reflejaran adecuadamente la forma en que el estigma relacionado con el VIH se expresa en el contexto local. Es decir, se procuró que los reactivos sean sensibles a la experiencia real de las personas con VIH del AMBA y, en consecuencia, puedan captar los niveles existentes de estigma.

Cada criterio debía ser respondido mediante una escala de 4 puntos, siendo 1 que el reactivo no cumple con el criterio, y 4, que cumple totalmente con él. Para cada uno de los cinco criterios se proveyeron indicadores detallados para su evaluación. Asimismo, se solicitó a los expertos que incluyeran todas las correcciones y observaciones cualitativas que estimasen necesarias, como complemento de la evaluación cuantitativa. Los expertos realizaron sus juicios de forma independiente.

Se analizó el grado de acuerdo entre jueces, mediante el cálculo del porcentaje de acuerdo. Se consideró que un ítem cumplía con un criterio cuando el $80 \%$ de los jueces acordaba que así era (o el $60 \%$ había optado por la opción 4 -cumple con el criterio- mientras que el $40 \%$ habia elegido la opción 3,-cumple moderadamente con el criterio-). Dadas las limitaciones estadísticas del porcentaje de acuerdo, se optó por calcular, de forma adicional, el coeficiente $V$ de Aiken para evaluar el grado de acuerdo entre 
jueces (Escurra, 1988). Se consideró que un item cumplía con un criterio cuando alcanzaba un $V$ de .80 .

Se contrastaron ambos cálculos. Aquellos enunciados que no cumplian con 3 criterios o más, para ambos cálculos, fueron eliminados. Los items que no cumplian con 1 o 2 criterios fueron modificados tomando como base las recomendaciones y observaciones de los jueces expertos. Sólo se halló discrepancia entre el porcentaje de acuerdo y el coeficiente $V$ de Aiken para 18 reactivos. De ellos, 8 fueron reformulados de acuerdo con observaciones de los jueces y 10 fueron mantenidos por motivos teóricos, a pesar de estar al límite del punto de corte del coeficiente $V$ de Aiken. Como resultado, se eliminaron 37 items del conjunto original y se mantuvieron 51. Asimismo, se agregaron 28 enunciados nuevos sugeridos por los jueces expertos por representar algún aspecto relevante o significativo del constructo que no había sido considerado en el conjunto original.

Por recomendación de los jueces, se realizaron correcciones en la consigna general y se modificaron las opciones de respuesta. Se incorporó una opción intermedia ("ni de acuerdo ni en desacuerdo"), por lo que la escala pasó a tener 5 opciones, en lugar de las 4 originales. El producto final de esta primera depuración fue una primera versión preliminar del IE-VIH de 79 ítems. El protocolo de respuestas resultante fue confeccionado ordenando los reactivos de manera tal que las escalas no se presentaran aisladas y que los items de cada una de ellas se presentaran intercalados.

\section{PRUEBA PILOTO}

\section{Participantes}

\section{Fase 1 de Etapa Piloto}

En la Fase 1 de la etapa de Prueba Piloto se administró la primera versión preliminar del IE-VIH, de 79 items, a una muestra no probabilística intencional de 10 personas con $\mathrm{VIH}$, con edades comprendidas entre los $27 \mathrm{y}$ los 56 años $(M=43.50 ; D E=7.51)$. Esta muestra estuvo conformada por 9 hombres y 1 mujer. Todos los participantes reportaron un nivel educativo equivalente a secundario completo o superior.

\section{Fase 2 de Etapa Piloto}

En la Fase 2 de la etapa de Prueba Piloto se empleó una muestra no probabilística conformada por 46 personas con VIH de ambos sexos (31 hombres, 14 mujeres y una mujer transgénero), con edades comprendidas entre 23 y 68 años $(M=41.4 ; D E=10.6)$. En la selección de esta muestra se excluyó a quienes habían participado de la Fase 1. E1 50\% de los participantes había sufrido al menos una experiencia de estigma y discriminación debida al VIH. El entorno cotidiano (familiares, amigos y parejas estables y ocasionales) fue el principal ámbito en el que los participantes informaron haber recibido estigma, rechazo y discriminación a causa de su diagnóstico $(32.6 \%$ de la muestra). Los otros dos ámbitos más frecuentemente mencionados como escenarios de episodios de estigma experimentado fueron el laboral (23.9\%) y servicios de salud $(26.1 \%)$. El resto de los datos descriptivos de esta muestra pueden apreciarse en la tabla 1. 
Tabla 1

Características de los participantes.

\begin{tabular}{|c|c|c|c|c|}
\hline Variable & $N$ & $\%$ & $M$ & $D E$ \\
\hline \multicolumn{5}{|l|}{ Género } \\
\hline Varón & 31 & 67.4 & & \\
\hline Mujer & 14 & 30.4 & & \\
\hline Mujer Transgénero & 1 & 2.2 & & \\
\hline Edad & & & 41.46 & 10.68 \\
\hline \multicolumn{5}{|l|}{ Nivel Educativo } \\
\hline Primario completo & 5 & 10.9 & & \\
\hline Secundario incompleto & 7 & 15.2 & & \\
\hline Secundario completo & 4 & 8.7 & & \\
\hline Terciario/Univ. incompleto & 11 & 23.9 & & \\
\hline Terciario/Univ. completo & 16 & 34.8 & & \\
\hline Posgrado & 3 & 6.5 & & \\
\hline \multicolumn{5}{|l|}{ Situación Laboral } \\
\hline Pensionado/Jubilado & 5 & 10.9 & & \\
\hline Desempleado & 9 & 19.6 & & \\
\hline Empleado Público/Privado & 22 & 47.8 & & \\
\hline Patrón/Cuentapropista & 10 & 21.8 & & \\
\hline \multicolumn{5}{|l|}{ Orientación Sexual } \\
\hline Heterosexual & 20 & 43.5 & & \\
\hline Homosexual & 22 & 47.8 & & \\
\hline Bisexual & 4 & 8.7 & & \\
\hline \multicolumn{5}{|l|}{ Vías de transmisión } \\
\hline Sexual & 33 & 71.7 & & \\
\hline Otras (transfusiones, transmisión vertical, drogas) & 4 & 8.7 & & \\
\hline No sabe/No recuerda & 9 & 19.6 & & \\
\hline Años en tratamiento & & & 6.64 & 6.84 \\
\hline \multicolumn{5}{|l|}{ Experiencia de Estigma por VIH } \\
\hline Sí & 23 & 50 & & \\
\hline No & 23 & 50 & & \\
\hline Entorno cotidiano (amigos, familia, pareja) & 15 & 32.6 & & \\
\hline Ámbito laboral & 11 & 23.9 & & \\
\hline Servicio de salud & 12 & 26.1 & & \\
\hline Institución educativa & 2 & 4.3 & & \\
\hline Institución religiosa & 2 & 4.3 & & \\
\hline Organismo gubernamental (ANSES, registro civil, etc.) & 2 & 4.3 & & \\
\hline Institución recreativa (baile, teatro, cine) & 1 & 2.2 & & \\
\hline
\end{tabular}

\section{Instrumentos}

Inventario de Estigma relacionado con el VIH (IE-VIH), versión preliminar. En la Fase 2 de la etapa de Prueba Piloto, se aplicó la segunda versión preliminar de la IE-VIH que constó de 77 ítems, con una escala de respuesta de 1 (muy en desacuerdo) a 5 (muy de acuerdo).

Escala de Estigma de VIH (EEV). La adaptación española de la versión revisada y reducida de la Escala de Estigma de VIH (HIV Stigma Scale, Berger et al., 2001; versión abreviada y revisada, Bunn et al., 2007; adaptación española, Fuster Ruiz, 2011) fue administrada en la Fase 2 de la etapa de Prueba Piloto con el objetivo de obtener evidencia de validez de criterio concurrente para el nuevo instrumento (IE-VIH). La escala, en su versión española, está compuesta por 30 items que evalúan el grado de estigma percibido en personas con VIH y que se responden con una escala Likert de 4 opciones, siendo 1 (totalmente en desacuerdo) y 4 (totalmente de acuerdo). Los reactivos de la escala en su versión española se agrupan en 4 factores, obtenidos por Análisis Factorial Exploratorio, que reproducen los de las versiones original y reducida: Estigma personal percibido (equivalente a Estigma experimentado), Auto-imagen negativa (equivalente a Estigma 
internalizado), Preocupaciones acerca de revelar la serología y Preocupación por las actitudes públicas de rechazo (ambos equivalentes a Estigma anticipado). Las dimensiones mencionadas han demostrado una adecuada confiabilidad por consistencia interna, con alfas de Cronbach que oscilan entre .70 y .88 (Fuster Ruiz, 2011).

Cuestionario sociodemográfico. Se aplicó un cuestionario sociodemográfico, construido específicamente para esta investigación, con el fin de evaluar variables sociodemográficas básicas (edad, género, nivel educativo, ocupación, estado civil, orientación sexual), variables específicas relacionadas con el VIH (año de recepción del diagnóstico, vía de transmisión, grado de revelación del diagnóstico a otros, cantidad de años en tratamiento) y variables relacionadas con el estigma experimentado (experiencia previa de estigma y en qué contextos aconteció).

\section{Procedimiento}

\section{Fase 1 de Etapa Piloto - Versión de 79 ítems}

Con el fin de realizar una primera prueba del instrumento con la población destinataria, se llevó a cabo un primer estudio piloto de menor escala. Se administró la versión preliminar del IE-VIH de 79 ítems a una muestra de 10 personas con VIH en formato de entrevista estructurada, para recabar información de tipo cualitativo. Los participantes recibieron la consigna de realizar todas las sugerencias, comentarios y observaciones que estimasen relevantes respecto del instrumento y las frases que lo componian. Se indicó que prestaran particular atención tanto a los aspectos formales (claridad de la redacción, simplicidad de los términos empleados, comprensión general de la consigna y los enunciados) como a los aspectos de contenido (por ejemplo, si las frases hacian referencia a situaciones que efectivamente experimentan las personas con VIH en el contexto local).

Debido a las características del constructo medido, que implica experiencias negativas de rechazo y discriminación, se evaluó específicamente el posible impacto emocional negativo de responder la prueba, mediante una pregunta al final de la entrevista.

La información cualitativa recolectada fue analizada y sistematizada. Tomando como referencia las observaciones y recomendaciones de los entrevistados, 2 ítems fueron eliminados debido a que su redacción resultó confusa para una proporción significativa de los sujetos. Esto dio como resultado una nueva versión preliminar del IE-VIH de 77 items. Los entrevistados concluyeron que, aunque responder al instrumento los remitía a situaciones y experiencias desagradables pasadas, no habian experimentado un impacto emocional negativo significativo.

\section{Fase 2 de Etapa Piloto - Versión de 77 ítems}

Se administró esta segunda versión preliminar de la prueba (77 ítems) a una muestra mayor de personas con VIH, junto con la Escala de Estigma de VIH y el cuestionario sociodemográfico.

Esta fase de la investigación tuvo la aprobación del Comité de Ética de la Organización No Gubernamental en la que se desarrolló el estudio. Los participantes fueron convocados a través de los y las profesionales que los atendian (médicos/as, psicólogas, otros) en la institución sede de esta 
investigación y a través de los distintos medios de contacto que esta posee. Fueron incluidas aquellas personas con VIH, mayores de 18 años, que espontáneamente expresaron interés en participar de este estudio. Todos los participantes fueron informados de los objetivos de esta investigación y firmaron un consentimiento escrito antes de iniciar su participación. Esta fue totalmente voluntaria y se garantizó la confidencialidad de las respuestas a lo largo de todo el proceso.

Las entrevistas de administración de los instrumentos fueron realizadas entre noviembre y diciembre de 2016. Los instrumentos fueron administrados como entrevistas estructuradas por el investigador a cargo, con el fin de explorar las reacciones de los sujetos al instrumento y registrar comentarios y observaciones de los entrevistados que pudieran resultar significativas.

\section{Análisis de datos}

\section{Análisis de los ítems}

A partir de los datos obtenidos en la Fase 2 de la etapa piloto, se realizó un análisis descriptivo de los items, examinando la media, desviación estándar, distribución de frecuencia, asimetría y curtosis de cada uno de ellos. Como criterio para evaluar los índices de asimetría y curtosis se consideraron como excelentes valores entre +1.00 y -1.00 , y como adecuados valores inferiores a +2.00 y -2.00 (George \& Mallery, 2011). Asimismo, se estudió la discriminación del ítem a partir del análisis de correlación de cada reactivo con el puntaje total de su dimensión correspondiente. Se consideró que los items con correlaciones no significativas o bajas con el puntaje total (inferiores a .30) debían ser eliminados o revisados.

\section{Fiabilidad y validez de criterio de las escalas}

Se exploraron las propiedades psicométricas de la versión preliminar del IE-VIH (de 77 items). En primer lugar, se realizó un análisis de consistencia interna (coeficiente alfa de Cronbach) de cada una de las escalas para evaluar la confiabilidad. Por otro lado, de acuerdo a Hogan (2004), un método para obtener evidencia de validez concurrente es demostrar la correlación entre la prueba a ser validada y algún otro instrumento reconocido como indicador válido del constructo objeto de estudio. Por esta razón, con el fin establecer la validez concurrente del IE-VIH, se consideraron las correlaciones entre cada dimensión y el factor de la adaptación española de la Escala de Estigma de VIH (versión abreviada), conceptualmente equivalente a ella.

\section{RESULTADOS}

\section{Análisis descriptivo de los ítems}

Con el objetivo de comprobar los supuestos de normalidad de la muestra, se realizaron análisis de asimetría y curtosis para cada item. Los resultados permitieron concluir que, del total de 77 items, 69 presentaban valores de asimetría y curtosis excelentes o adecuados. Por el contrario, 8 items presentaron valores inadecuados y fueron eliminados.

En relación con el análisis de discriminación, la mayor parte de los reactivos (54) arrojó valores superiores al punto de corte establecido (.30) en 
las correlaciones item-total corregidas. En contraste, 23 items presentaron valores inferiores $\mathrm{y}$, en consecuencia, fueron descartados, cuidando que la eliminación no afectara negativamente el alfa de Cronbach de cada escala. Adicionalmente, 4 ítems fueron eliminados, a pesar de contar con adecuada asimetría, curtosis y superar el punto de corte para la correlación item-total. El motivo es que fueron considerados redundantes, poco significativos y/o ajenos a las experiencias reales de los participantes, según los comentarios y observaciones de estos, registradas durante la fase de administración.

En conclusión, un total de 27 reactivos de la primera versión preliminar del IE-VIH fueron descartados, dando como resultado una nueva versión depurada de 50 ítems. La escala de Estigma Anticipado quedó conformada por 18 items, mientras que las escalas de Estigma Experimentado y Estigma Internalizado quedaron constituidas por 16 ítems cada una.

\section{Análisis de confiabilidad}

Para establecer la consistencia interna del IE-VIH, se calcularon los coeficientes alfa de Cronbach de cada escala. Todos los valores resultaron superiores a .85, considerados buenos por la literatura (George \& Mallery, 2011; Nunnally, 1978). En la tabla 2 se reportan los coeficientes obtenidos para cada escala, junto con las medias y desvíos estándar.

Tabla 2

Medias, desvíos estándar y alfas de Cronbach de las Escalas del IE-VIH.

\begin{tabular}{lccc}
\hline Variable & $\boldsymbol{M}$ & $\boldsymbol{D E}$ & Alfa de Cronbach \\
\hline Estigma Anticipado & 58.54 & 13.72 & .85 \\
Estigma Internalizado & 41.85 & 13.02 & .86 \\
Estigma Experimentado & 33.76 & 13.19 & .86 \\
\hline
\end{tabular}

\section{Validez concurrente: correlaciones con la Escala de Estigma de VIH}

Con el fin de obtener evidencia de validez concurrente, se analizaron las correlaciones entre las tres escalas del IE-VIH (versión depurada de 50 ítems) y los cuatro factores de la Escala de Estigma de VIH. Se exploraron las asociaciones entre "Estigma experimentado" y el factor "Estigma personal percibido", "Estigma internalizado" y el factor "Auto-imagen negativa" y "Estigma anticipado" y los factores "Preocupaciones acerca de revelar la serología y "Preocupación por las actitudes públicas de rechazo". Los resultados obtenidos indican la existencia de asociaciones positivas significativas entre las escalas de ambos instrumentos que son conceptualmente equivalentes entre sí, de acuerdo con lo esperado. Los coeficientes de correlación obtenidos pueden ser apreciados en la tabla 3.

\section{Descripción de los niveles de estigma relacionado con el VIH}

En la tabla 2, se pueden apreciar las medias y desvíos estándar para cada escala del IE-VIH. El mecanismo de estigma predominante entre los participantes fue el Estigma Anticipado, seguido por el Internalizado y, en último lugar, el Experimentado. 
Tabla 3

Correlaciones de Pearson entre las Escalas del IE-VIH y los Factores de la Escala de Estigma de VIH (EEV), versión abreviada, adaptación española.

\begin{tabular}{lc}
\hline Variable & $\begin{array}{c}\text { rde } \\
\text { Pearson }\end{array}$ \\
\hline IE-VIH: Estigma Internalizado / EEV: Autoimagen negativa & $.68^{* *}$ \\
IE-VIH: Estigma Anticipado / EEV: Preocupaciones acerca de revelar la serología & $.81^{* *}$ \\
IE-VIH: Estigma Anticipado / EEV: Preocupaciones por las actitudes públicas de rechazo & $.47^{* *}$ \\
IE-VIH: Estigma Experimentado / EEV: Estigma personal percibido & $.79^{* *}$ \\
\hline${ }^{* *} p<.01$ &
\end{tabular}

\section{DISCUSIÓN}

En el presente trabajo se presentan los avances en la construcción de un instrumento para evaluar el estigma percibido por las personas con VIH adultas del AMBA: el IE-VIH. La construcción de esta prueba responde a la necesidad de contar con un instrumento adecuado al contexto sociocultural, actualizado en relación con la realidad presente de la epidemia y sensible a las experiencias efectivamente vividas por las personas con $\mathrm{VIH}$, para medir esta variable con fines de investigación e intervención.

Respecto de las propiedades psicométricas, los análisis de la primera versión preliminar demuestran que el IE-VIH posee adecuada confiabilidad por consistencia interna. Asimismo, se han efectuado los procedimientos metodológicos para asegurar que el muestreo de los reactivos sea representativo del constructo a medir y de sus dimensiones teóricas, garantizando la validez de contenido para el IE-VIH. Adicionalmente, también se obtuvo evidencia en favor de la validez concurrente de este instrumento, a través de la correlación de sus puntuaciones con las de otra prueba conceptualmente equivalente: la Escala de Estigma de VIH. Es posible concluir que la versión preliminar del IE-VIH ha demostrado poseer aceptables propiedades psicométricas, dando cuenta de un instrumento promisorio. Partiendo de una versión preliminar psicométricamente adecuada es posible anticipar que existen mayores probabilidades de arribar a una versión final igualmente apropiada.

Respecto de la validez, en particular, adherimos a lo propuesto por Escobar y Cuervo (2008) en el sentido de que esta es una propiedad que siempre está en relación con el propósito, el contexto y los destinatarios para los que un instrumento está siendo construido o adaptado. Desde el inicio del proceso de construcción, se implementaron procedimientos para garantizar la adecuación del IE-VIH al contexto local en que será utilizado, considerando sus particularidades socio-culturales. Asimismo, se incluyó la perspectiva de los destinatarios tratando de asegurar que los items del inventario reflejen las percepciones y emociones de las personas con VIH del AMBA y las experiencias que efectivamente viven con relación al estigma. Es esperable que esto redunde en un instrumento más sensible para la detección del estigma percibido y experimentado por esta población específica.

Por otro lado, los resultados arrojados por esta versión preliminar son consistentes con los proporcionados por estudios previos realizados en Argentina, como el de Fundación Huésped (2012). Ambos estudios coinciden en el amplio predominio del estigma interno (internalizado y anticipado) por sobre el externo o experimentado en personas con VIH en este país. Una explicación posible es que esto puede deberse a cambios en la expresión del 
estigma que acompañan la evolución de la epidemia. Un mayor conocimiento científico acerca del VIH y la existencia de campañas preventivas e informativas periódicas puede haber impactado positivamente en el componente cognitivo-informacional del estigma relacionado con el VIH, desalentando sus expresiones más abiertas y extremas. La mayor divulgación de información acerca del VIH y sus vías de transmisión, así como el refuerzo de la norma social y legal de no discriminación, puede haber influido en la reducción de expresiones públicas de estigma. No obstante, sus formas más sutiles y encubiertas, pertenecientes al ámbito privado de los individuos y relacionadas a prejuicios preexistentes, pueden persistir, manteniendo su impacto negativo (Herek et al., 2002). Posiblemente, esto explique el hecho de que las personas con VIH refieran un menor nivel de experiencias efectivas de estigma a la vez que mantienen el temor y la expectativa de que esas experiencias puedan ocurrir en algún momento e incorporen en su autoconcepto los prejuicios y sentimientos negativos que perciben en la sociedad respecto de ellos mismos. La exploración de esta hipótesis puede considerarse un punto relevante de ser abordado en futuras investigaciones sobre este fenómeno en Argentina.

Los resultados presentados en este trabajo constituyen una muestra parcial de un proceso que continúa su avance. En este sentido, futuras etapas incluirán la realización de análisis factoriales, tanto exploratorios como confirmatorios, con el fin de poner a prueba la estructura tridimensional propuesta y aportar evidencia de validez de constructo para el inventario. Esto implicará el empleo de muestras de participantes más grandes y representativas, en relación con la información epidemiológica actualmente disponible respecto del VIH en el AMBA. Una muestra más amplia permitirá, asimismo, realizar nuevos estudios acerca de la confiabilidad de las escalas.

Adicionalmente, en una próxima etapa, se planea administrar el IE-VIH conjuntamente con alguna medida de deseabilidad social. El estigma relacionado con el VIH remite, en todos los casos, a experiencias pasadas desagradables y emociones displacenteras. El estigma internalizado, en especial, involucra emociones como culpa y vergüenza que los sujetos pueden estar menos dispuestos a reconocer frente a otros, dadas las características sociales intrinsecas de estos estados emocionales. Por otro lado, algunas experiencias específicas de rechazo o agresión vividas (por ejemplo, el rechazo de eventuales parejas sexuales) o algunas afirmaciones referidas a oportunidades futuras (como, por ejemplo, que el VIH ha limitado proyectos futuros) pueden ser dificiles de aceptar y comunicar por los propios sujetos, dado que afectan el autoconcepto. Además, su reconocimiento frente a otros (como el entrevistador), puede dejar a quien responde particularmente expuesto, desencadenando sentimientos de vergüenza. Por estos motivos, los individuos estigmatizados pueden tender a negar o minimizar el prejuicio y la discriminación con el fin de proteger su autoestima (Shih, 2004). De esto se desprende una mayor probabilidad de que los sujetos distorsionen sus respuestas, conscientemente o no, lo que puede introducir sesgos que afecten significativamente las puntuaciones y reduzcan su confiabilidad. En este sentido, se recomienda combinar el IE-VIH con alguna medida de deseabilidad social, con el fin de evaluar el impacto de esta variable en las respuestas de los sujetos.

Resulta interesante destacar, finalmente, algunos aportes no esperados del instrumento que se deducen de los comentarios y observaciones de los 
participantes durante las entrevistas de administración. Por un lado, los sujetos coincidieron en que no habian experimentado un impacto emocional negativo significativo al responder al IE-VIH, aun cuando los enunciados los remitian a experiencias desagradables del pasado y a emociones displacenteras. Por otro lado, una porción significativa refirió, como elemento positivo, que responder al instrumento le había permitido reflexionar sobre aspectos de su condición de salud que no había considerado con anterioridad o sobre los que no había tomado conciencia. Este puede resultar un aporte adicional interesante de ser explorado en investigaciones futuras, acerca de usos no convencionales de los instrumentos de evaluación. En el marco de una intervención clínica, el IE-VIH no solo puede tener su utilidad evaluativa original, sino también puede ser el disparador de un proceso reflexivo sobre distintos aspectos del hecho de vivir con VIH, en el marco de una intervención psicoterapéutica.

La versión preliminar del IE-VIH resulta un avance prometedor de cara a la construcción de un nuevo instrumento para medir estigma relacionado con el VIH. Una herramienta localmente construida, válida, confiable y que refleje las particularidades del contexto para el que está destinada resulta un aporte sustancial en diversos sentidos. En primer lugar, permite contar con un instrumento para la investigación de este fenómeno en el AMBA y en Argentina. De este modo, se espera que promueva un mejor y más profundo conocimiento del fenómeno del estigma relacionado con el VIH, en general, y en Argentina, en particular. Contar con una mejor descripción de como se expresa el estigma en nuestro contexto y contar con un acervo mayor de información disponible al respecto permitirá orientar con mayor precisión el diseño de intervenciones de reducción de estigma en el futuro. Adicionalmente, no solo es un aporte al diseño de las intervenciones sino también a la evaluación de su eficacia y, en última instancia, al mejoramiento del bienestar y la calidad de vida de las personas con VIH.

\section{REFERENCIAS}

Baral, S., Logie, C. H., Grosso, A., Witz, A. L., \& Beyrer, C. (2013). Modified social ecological model: a tool to guide the assessment of the risks and risk contexts of HIV epidemics. BMC Public Health, 13, 482-490. https://doi.org/10.1186/1471-2458-13-482

Bauer, G. R. (2014). Incorporating intersectionality theory into population health research methodology: Challenges and the potential to advance health equity. Social Science \& Medicine, 110, 10-17. https://doi.org/10.1016/j.socscimed.2014.03.022

Berger, B. E., Ferrans, C. E., \& Lashley, F. R. (2001). Measuring stigma in people with HIV: Psychometric assessment of the HIV stigma scale. Research in Nursing and Health, 24, 518-529. https://doi.org/10.1002/nur.10011

Bilder, P. (2010). "Una visita inesperada". Primeros años del SIDA en la Argentina (1981-1984). En H. Vessuri, P. Kreimer, A, Arellano \& L. Sanz Méndez (Eds.), Conocer para transformar: Producción y reflexión sobre ciencia, tecnología e innovación en Iberoamérica (pp. 35-53). Caracas: UNESCO-IESALC.

Bunn, Y. J., Solomon, S. E., Miller, C., \& Forehand, R. (2007). Measurement of stigma in people with HIV: A reexamination of the HIV Stigma Scale. AIDS Education and Prevention, 19(3), 198-208. doi: 10.1521/aeap.2007.19.3.198 https://doi.org/10.1521/aeap.2007.19.3.198

Chambers, L. A., Rueda, S., Baker, D. N., Wilson, M. G., Deutsch, R., Raeifar, E., ... The Stigma Review Team. (2015). Stigma, HIV and health: A qualitative synthesis. BMC Public Health, 15, 1-17. https://doi.org/10.1186/s12889-015-2197-0

Crenshaw, K. (1989). Demarginalizing the intersection of race and sex: A black feminist critique of antidiscrimination doctrine, feminist theory and antiracist politics. University of Chicago Legal Forum, 1989(1), 139-167.

Crocker, J., Major, B., \& Steele, C. (1998). Social Stigma. En D.T. Gilbert, S.T Fiske \& G. Lindzey (Eds.), The Handbook of social psychology ( $4^{\mathrm{a}}$ ed., pp. 504-553). New York: McGraw-Hill.

Deacon, H. (2006). Towards a sustainable theory of health-related stigma: Lessons from the HIV/AIDS literature. Journal of Community \& Applied Social Psychology, 16, 418-425. https://doi.org/10.1002/casp.900

Earnshaw, V. A., Lang, S. M., Lippitt, M., Jin, H., \& Chaudoir, S. R. (2015). HIV stigma and physical health 
symptoms: do social support, adaptive coping, and/or identity centrality act as resilience resources? AIDS and Behavior, 19, 41-49. https://doi.org/10.1007/s10461-014-0758-3

Earnshaw, V. A., Smith, L. R., Chaudoir, S. R., Rivet Amico, K., \& Copenhaver, M. M. (2013). HIV stigma mechanisms and well-being among PLWH: A test of the HIV stigma framework. AIDS and Behavior, 17(5), 1785-1795. https://doi.org/10.1007/s10461-013-0437-9

Escobar-Pérez, J. \& Cuervo-Martínez, A. (2008). Validez de contenido y juicio de expertos: Una aproximación a su utilización. Avances en Medición, 6, 27-36.

Escurra, L. M. (1988) Cuantificación de la validez de contenido por criterio de jueces. Revista de Psicología de la Pontificia Universidad Católica del Perú, 6(1-2), 103-111.

Franke, M. F., Nelson, A. K., Muñoz, M., Santa Cruz, J., Atwood, S., Lecca, L., \& Shin, S.S. (2014). Validation of 2 Spanish-language scales to assess HIV-related stigma in communities. Journal of the International Association of Providers of AIDS Care, 1-9. https://doi.org/10.1177/2325957414547738

Fundación Huésped (2012). Percepciones y experiencias sobre estigma y discriminación en poblaciones trans, HSH, y usuarios de drogas. Buenos Aires: Autor. https://doi.org/10.13140/RG.2.1.2803.0167

Fuster Ruiz, M. J. (2011). La percepción del estigma en las personas con VIH: sus efectos y formas de afrontamiento. Tesis de Doctorado, Departamento de Psicología Social y de las Organizaciones, Facultad de Psicología, Universidad Nacional de Educación a Distancia, Madrid, España.

George, D. \& Mallery, P. (2011). SPSS for Windows step by tep: A simple guide and reference. 18.0 update (1ra. Ed.). Boston: Allyn and Bacon.

Goffman, E. (1963). Estigma. La identidad deteriorada. Buenos Aires-Madrid: Amorrortu.

Hatzenbuehler, M. L., Phelan, J. C., \& Link, B. G. (2013). Stigma as a fundamental cause of population health inequalities. American Journal of Public Health, 103(5), 813-821. https: / / doi.org/ 10.2105/AJPH.2012.301069

Herek, G. M., Capitanio, J. P., \& Widaman, K. F. (2002). HIV-Related stigma and knowledge in the United States: Prevalence and trends, 1991-1999. American Journal of Public Health, 92(3), 371-377. https://doi.org/10.2105/AJPH.92.3.371

Herek, G. M., Saha, S., \& Burack, J. (2013). Stigma and psychological distress in people with HIV/AIDS. Basic and Applied Social Psychology, 35, 41-54. https://doi.org/10.1080/01973533.2012.746606

Hogan, T. (2004). Pruebas psicológicas: Una introducción práctica. Mexico: Manual Moderno Editorial.

Holzemer, W. L., Uys, L. R., Chirwa, M. L., Greeff, M., Makoae, L. N., Kohi, T. W.,... Durrheim, K. (2007) Validation of the HIV/AIDS stigma instrument - PLWA (HASI-P). AIDS Care, 19(8), 1002-1012 https://doi.org/10.1080/09540120701245999

Jeffries, W. L., Townsend, E. S., Gelaude, D. J., Torrone, E. A., Gasiorowicz, M., \& Bertolli, J. (2015). HIV stigma experienced by young men who have sex with men (MSM) living with HIV infection. AIDS education and prevention, 27(1), 58-71. https://doi.org/10.1521/aeap.2015.27.1.58

Jones, E. E., Farina, A., Hestrof, A. H., Markus, H., Miller, D. T., \& Scott, R. A. (1984). Social stigma: The psychology of marked relationships. New York: Freeman.

Kipp, A. M., Audet, C. M., Earnshaw, V., Owens, J., McGowan, C. C., \& Wallston, K. A. (2015). ReValidation of the Van Rie HIV/AIDS-Related Stigma Scale for Use with People Living with HIV in the United States. PLoS One, 10(3). https://doi.org/10.1371/journal.pone.0118836

Kurzban, R. \& Leary, M. R. (2001). Evolutionary origins of stigmatization: The functions of social exclusion. Psychological Bulletin, 127(2), 187-208. https://doi.org/10.1037/0033-2909.127.2.187

Link, B. G. \& Phelan, J. C. (2001). Conceptualizing stigma. Annual Review of Sociology, 27, 363-385. https://doi.org/10.1146/annurev.soc.27.1.363

Link, B. G. \& Phelan, J. C. (2006). Stigma and its public health implications. The Lancet, 367, 528-529. https: / /doi.org/10.1016/S0140-6736(06)68184-1

Logie, C. \& Gadalla, T. M. (2009). Meta-analysis of health and demographic correlates of stigma towards people living with HIV. AIDS Care, 21(6), 742-753. https://doi.org/10.1080/09540120802511877

Mahajan, A. P., Sayles, J. N., Patel, V. A., Remien, R. H., Ortiz, D., Szekeres, G., \& Coates, T. J. (2008). Stigma in the HIV/AIDS epidemic: A review of the literature and recommendations for the way forward. AIDS, 22(2), 67-79. https://doi.org/10.1097/01.aids.0000327438.13291.62

Matesanz Nogales, A. (1997). Evaluación estructurada de la personalidad. Madrid: Pirámide.

Molina, Y. \& Ramirez-Valles, J. (2013). HIV/AIDS stigma: Measurement and relationships to psychobehavioral factors in Latino gay/bisexual men and transgender women. AIDS Care, 1-10. https://doi.org/10.1080/09540121.2013.793268

Nunnally, N. C. (1978). Psychometric Theory. New York: McGraw-Hill.

Nyblade, L. C. (2006). Measuring HIV Stigma: Existing knowledge and gaps. Psychology, Health and Medicine, 11(3), 335-345. https://doi.org/10.1080/13548500600595178

Ogden, J. \& Nyblade, L. (2005). Common at its core: HIV-related stigma across contexts. International Center for Research on Women (ICRW): Washington D.C.

Parker, R. \& Aggleton, P. (2003). HIV and AIDS-related stigma and discrimination: a conceptual framework and implications for action. Social Science and Medicine, 57, 13-24. https://doi.org/10.1016/S02779536(02)00304-0

Pecheny, M. (2008). Estudio sobre la situación social de las Personas Viviendo con VIH en la Argentina. Buenos Aires: UBATEC.

Pecheny, M., Manzelli, H. M., \& Jones, D. E. (2007). The experience of stigma: People living with HIV/AIDS and Hepatitis $\mathrm{C}$ in Argentina. Revista Interamericana de Psicologia/ Interamerican Journal of Psychology, 41(1), 17-30.

Phelan, J. C., Link, B. G., \& Dovidio, J. F. (2008). Stigma and prejudice: One animal or two? Social Science \& Medicine, 67, 358-367. https://doi.org/10.1016/j.socscimed.2008.03.022

Rosas Torres, A. R. \& Gomes Verissimo de Faria, M. R. (2008). Creencia en un mundo justo y prejuicios: el 
caso de los homosexuales con VIH/SIDA. Interamerican Journal of Psychology, 42(3), 570-579.

Sayles, J. N., Hays, R. D., Sarkisian, C. A., Mahajan, A. P., Spritzer, K. L., \& Cunningham, W. E. (2008). Development and psychometric assessment of a multidimensional measure of internalized HIV stigma in a sample of HIV-positive adults. AIDS and Behavior, 12, 748-758. https://doi.org/10.1007/s 10461008-9375-3

Shih, M. (2004). Positive stigma: Examining resilience and empowerment in overcoming stigma. The ANNALS of the American Academy of Political and Social Science, 591, 175-185. https://doi.org/10.1177/0002716203260099

Sowell, R. L., Lowenstein, A., Moneyham, L., Demi, A., Mizuno, Y., \& Seals, B. F. (1997). Resources, stigma, and patterns of disclosure in rural women with HIV infection. Public Health Nursing, 14(5), 302-312. https://doi.org/10.1111/j.1525-1446.1997.tb00379.x

Visser, M. J., Kershaw, T., Makin, J. D., \& Forsyth, B. W. C. (2008). Development of parallel scales to measure HIV-related stigma. AIDS Behavior, 12, 759-771. https://doi.org/10.1007/s10461-008-93637

Recibido 20-04-2017 | Aceptado 17-07-2017 